\title{
Editorial: Mechanics of Legged Robots: From Bio-Inspiration to Novel Legged Machines
}

\author{
Giovanni Gerardo Muscolo ${ }^{1 *}$ and Marco Ceccarelli ${ }^{2 *}$ \\ ${ }^{1}$ Department of Computer Science, University of Verona, Verona, Italy, ${ }^{2}$ Laboratory of Robot Mechatronics, DII-Department \\ of Industrial Engineering, University of Rome Tor Vergata, Rome, Italy
}

Keywords: legged robots, mechanics of locomotion, biomimetic design, biped robots, exoskeletons, walking, running, balance

\section{Editorial on the Research Topic}

\section{Mechanics of Legged Robots: From Bio-Inspiration to Novel Legged Machines}

Balance and locomotion performances in legged robots are two crucial aspects that are usually optimized by using novel algorithms and control architectures. On the contrary, in this Research Topic, the central role for increasing robot performance is focused to mechanics of walking machines, by proposing novel models of legged robots with an optimized mechanics as bio-inspired from nature.

One goal of this special issue is to outline how performance of legged robots may be designed and improved by using advanced mechanics knowledge. The success of the topic is underlined by the following published works all in symbiosis with the main idea at using a simple mechanism to control the behavior of a complex system in robotic locomotors.

In Maiorino and Muscolo a simple compliant mechanism is included in the lower leg joints (ankle, knee, hip) of a multibody human model, discovering how the appropriate calibration of the compliance of the system may increase the robot performance in walking and running, without modifying the power supply requested by actuators. These extraordinary results confirm the importance of the research field lunched with this Research Topic.

The mechanism proposed in Fisher et al. allowed to conceive a robot, named Baleka, with a rapid maneuverability. A comparison with the developed robots in literature underlines the very good performance that is obtained with this robot. Experiments confirm the design feasibility of the mechanism for the legs and the entire robot. The only two points of contact with the ground of the Baleka robot are sufficient to test successfully locomotion and jumping operations.

In Tracuzzi Spadaro and Muscolo, the role of the compliance in a four legged robot is investigated for reducing power supply and increasing robot performance. Results underline how the compliance of the spine has a fundamental role for reducing gaps of power request from anterior to posterior legs (and vice versa) in four legged robots.

The work of Trono et al. presents a completely novel exoskeleton design, including actuated wheels in feet for supporting people in a complete locked-in state. The paper shows how with an appropriate mechanical design also market components like wheels it is possible to produce a ground breaking evolution in exoskeleton design and locomotion, which may be used for helping people with a more sustainable machine.

Driessen and Orsolino explore the shock propagation of impacts in rigid body systems. They revise the concept of the center of percussion and propose a newly derived variable called the radius of percussion. The novelty of the paper is focused on the investigation of the impact with an unconventional approach, which does not require the full knowledge of bodies' stiffness. This 
method may be complementary to classical analysis, and it opens up a new point of view to define shock impacts in legged robots.

An interesting investigation of how the biped locomotion may be performed with one actuator is presented in Zhang and Arakelian, where three mechanisms are proposed for novel solution. The kinematics of a single degree-of-freedom mechanism is discussed in details analyzing fundamental mechanism applied to biped locomotion namely fourbar mechanism, slider-crank mechanism, cam system, and pantograph mechanism.

In Mummolo et al., the balance of biped systems is proposed with a novel methodology approach to the problem of reproducing the human ability of keeping locomotion balance. In particular, general biped model balance capabilities are analyzed using the segmented feet approach so that the intermittent heel, flat, and toe contacts enable a contact model that maps discrete contact modes into corresponding center of pressure constraints.

\section{AUTHOR CONTRIBUTIONS}

GM was responsible for editing the papers Baleka: A Bipedal Robot for Studying Rapid Maneuverability and Design and Synthesis of Single-Actuator Walking Robots via Coupling of Linkages. MC was responsible for editing the papers Improving
Robustness of Legged Robots Against Mechanical Shock Using Impulsive Dynamics and State-Space Characterization of Balance Capabilities in Biped Systems with Segmented Feet. All authors contributed to the article and approved the submitted version.

\section{ACKNOWLEDGMENTS}

The Research Topic of Mechanics of Legged Robots: From BioInspiration to Novel Legged Machines has been launched by the following two Journals: Frontiers in Mechanical Engineering (section: Mechatronics) and Frontiers in Robotics and AI (section: Humanoid Robotics). A special thanks to the reviewers of the papers, which helped the editors to increase the quality of the Research Topic.

Conflict of Interest: The authors declare that the research was conducted in the absence of any commercial or financial relationships that could be construed as a potential conflict of interest.

Copyright (๑) 2021 Muscolo and Ceccarelli. This is an open-access article distributed under the terms of the Creative Commons Attribution License (CC BY). The use, distribution or reproduction in other forums is permitted, provided the original author(s) and the copyright owner(s) are credited and that the original publication in this journal is cited, in accordance with accepted academic practice. No use, distribution or reproduction is permitted which does not comply with these terms. 